\title{
SARS-CoV2 como causa de sepsis neonatal tardia, reporte de un caso
}

\section{SARS-CoV2 as a cause of late neonatal sepsis, a case report}

\author{
Delia-Edith Theurel-Amrtin ${ }^{1}$; Andrea Gomez ${ }^{1}$; Daniel Saldarriaga ${ }^{1}$; Katty-C Villegas-Lamus ${ }^{2}$; Jorge-Luis Alvarado-Socarras ${ }^{1}$
}

Forma de citar: Theurel-Amrtin DE, Gomez A, Saldarriaga D, Villegas-Lamus KC y Alvarado-Socarras JL. SARS-CoV2 como causa de sepsis neonatal tardía, reporte de un caso. Salud UIS. 2020; 53(4): 456-460.

doi: https://doi.org/10.18273/revsal.v52n4-2020013 (c) (i)

\begin{abstract}
Resumen
Introducción: la infección por SARS Cov2 en los niños tiene un comportamiento clínico menos severo que en adultos y por lo tanto menor mortalidad. Esta infección puede afectar neonatos y estos pueden adquirir la infección por trasmisión horizontal, con manifestaciones clínicas variables. Si bien, el neonato hijo de madre positiva para SARS CoV 2 ha sido el de mayor foco de atención, otros neonatos pueden adquirir la infección por casos positivos domiciliarios, sintomáticos o no. Reporte del caso: recién nacido previamente sano, de 22 días con manifestaciones sistémicas como fiebre, síntomas respiratorios leves y deposiciones liquidas frecuentes, sin moco ni sangre. Sin antecedentes de infecciones en la familia. El tratamiento fue sintomático, con acetaminofén, líquidos endovenosos y con evolución adecuada. Los laboratorios fueron inespecíficos, y se realizó estudio para SARS Cov2 por la pandemia actual. El reporte fue positivo y la familia rechazó realizarse pruebas. Discusión: la infección neonatal por SARS-CoV2 ha sido relacionada con síntomas como fiebre, cuadros respiratorios y gastrointestinales leves, generalmente con noción de contagio de personas sintomáticas o asintomáticas, que pueden ser subestimados. Es posible que el diagnóstico neonatal, sea la señal de adultos asintomáticos. Actualmente, ante los reportes literarios y la pandemia actual, se deberían realizar pruebas a neonatos febriles y con otros síntomas sistémicos. Además de realizar seguimiento a los neonatos afectados.
\end{abstract}

Palabras clave: SARS CoV 2; Fiebre; Trasmisión de SARS CoV 2; Neonato.

\begin{abstract}
Introduction: SARS Cov2 infection in children has a clinical evolution not as severe as in adults and therefore lower mortality. This infection can affect neonates and they can acquire the infection by horizontal transmission, with variable clinical manifestations. Although the neonate born to a mother positive for SARS-CoV 2 has been the one with the greatest focus of attention, other neonates can acquire the infection from positive cases at home, symptomatic or not. Case report: A 22-day-old, previously healthy newborn with systemic manifestations such as fever, mild respiratory symptoms and frequent liquid stools, without mucus or blood. No history of infections in the family. The treatment was symptomatic, with acetaminophen, intravenous fluids and with good evolution.

1. Fundación Cardiovascular de Colombia. Floridablanca. Santander.

2. Universidad Autónoma de Bucaramanga. Santander, Colombia

Correspondencia: Jorge Luis Alvarado Socarras. Dirección: Calle 155A 23-58. Urbanización El Bosque. Teléfono: +76399292 . Correo electrónico: Jorgealso2@yahoo.com
\end{abstract}


Laboratories were nonspecific, and the study for SARS Cov2 was realized due to the current pandemic. The report was positive and the family refused to carry out tests. Discussion: Neonatal SARS CoV2 infection has been associated with symptoms such as fever, mild respiratory and gastrointestinal symptoms, usually with a positive notion of contagion from symptomatic or asymptomatic people, that can be underestimated.. It is possible that the diagnosis in a neonate could be the alarm on asymptomatic adults. Currently, due to the reported cases and the SARS $\mathrm{CoV} 2$ pandemic, newborns with fever and other systemic symptoms should be tested in the neonatal period. On the other hand, perform a follow-up of affected newborns.

Keywords: SARS CoV 2; Fever; SARS CoV 2 transmission; Neonate.

\section{Introducción}

Los Coronavirus humanos (HCoVs) se han identificado desde 1960. El SARS- CoV2 (Severe acute respiratory syndrome coronavirus 2), asociado con la pandemia actual, pertenece a este grupo y fue identificado en Wuhan, China en diciembre del 2019. Estos virus han sido identificados principalmente como patógenos respiratorios, con cuadros clínicos variables desde síntomas como resfriado común, hasta neumonía grave y muerte. Sin embargo, también puede haber compromiso de otros sistemas como gastrointestinal y neurológico ${ }^{1}$. La enfermedad por SARS-CoV2 afecta a todos los grupos etarios, incluyendo gestantes, niños y recién nacidos; en este último grupo, existen discusiones acerca de la vía de trasmisión. Una es la forma vertical, la cual ha intentado ser demostrada desde el inicio de la pandemia, y con dos reportes recientes que potencialmente dejan abierta esta posibilidad ${ }^{2,3}$. La otra forma, es la trasmision horizontal, de las cuales existen multiples reportes de casos y cuya forma de adquisición podría ser contacto con madre positiva para SARSCoV2, personal de salud o contactos domiciliarios sintomáticos o asintomáticos.

A nivel mundial, los casos pediátricos reportados oscilan entre $0,8-2 \%$, de los cuales los casos reportados en menores de un año estan por debajo del $20 \%$. En Colombia, según datos del Instituto Nacional de Salud (INS), los menores de 9 años son el 3,6\% ${ }^{4}$, grupo con bajo porcentaje de mortalidad, donde la condición de prematurez y comorbilidades podrían jugar un papel importante 5 .

En Colombia, hasta la semana epidemiológica 31 (agosto 6), habían reportes de 270 casos neonatales $(0,1 \%)$, del total de casos reportados, con registro de una muerte, al parecer no relacionada con la infección ${ }^{4}$. Sin embargo, es probable subdiagnóstico por un cuadro clínico inespecífico y poco severo en este grupo etario, al menos en neonatos a término sin comorbilidades asociadas.
Presentamos el caso de un neonato a término, con síntomas muy inespecíficos y sin contacto epidemiológico aparente, detectado más como una pezquiza epidemiológica que por una verdadera intuición clínica.

\section{Reporte del Caso}

Producto de madre de 24 años, G2A1C1V1, con embarazo controlado sin alteraciones. Parto por cesárea en semana 39, con adaptación adecuada, peso $3.600 \mathrm{~g}$, talla $51 \mathrm{cms}$. Egresa sin complicaciones, con lactancia materna y fórmula de inicio. Al día 22, acude a consulta externa porque "le sonaba el pecho", niega otros síntomas como rinorrea, tos o fiebre. Formulan antihistamínico y lo egresan. 48 horas después, presentó quejido durante el sueño y fiebre de $38,2^{\circ} \mathrm{C}$, por lo cual le suministran acetaminofén. Por persistencia de fiebre consulta a urgencias a clínica de tercer nivel de atención. A su ingreso presentó temperatura de $37,7^{\circ} \mathrm{C}$ y leves tirajes subcostales. En el interrogatorio documentan antecedentes de síntomas respiratorios leves en madre y abuela. Padre del menor es asintomático, trabaja como domiciliario y fuera del núcleo familiar. Por los datos clínicos, sospechan sepsis neonatal, y hospitalizan. Toman hemograma, con leucocitos 8900 , neutrófilos $47 \%$, linfocitos $36 \%$, hemoglobina: $12,1 \mathrm{gr} / \mathrm{dl}$, hematocrito: $39 \%$, plaquetas: 356.000 , proteína $\mathrm{C}$ reactiva $48 \mathrm{mg} / \mathrm{L}$, electrolitos y uroanálisis normales. Remiten a la unidad de cuidados intensivos neonatales, por sospecha de sepsis por SARS-CoV2. A su ingreso la madre niega síntomas respiratorios en el recién nacido, refiere deposiciones líquidas, hasta 8 veces al día. Niega síntomas sugestivos en los contactos estrechos del neonato. Al ingreso buen estado general, frecuencia cardiaca de 159 latidos por minuto, frecuencia respiratoria 49 por minuto, tensión arterial 53/33 (percentil 25), oximetría 98\% sin oxígeno suplementario, peso $4,5 \mathrm{~kg}$. Sin alteraciones al examen físico. Se hospitaliza para vigilancia en área de aislamiento de contacto y respiratorio en incubadora, y con aporte enteral pleno por succión con leche materna 
extraída manualmente. Se solicita RT-PCR para SARSCoV-2 en hisopado orofaríngeo/nasofaríngeo, por antecedentes de sitio de remisión y por la situación epidemiológica, no por condición clínica del neonato. Se toman a las 24 horas de su ingreso laboratorios de control, proteína $\mathrm{C}$ reactiva de alta precisión de $2,47 \mathrm{mg} / \mathrm{dl}(0,0-5,0)$, hemograma con leucocitos 5980, hemoglobina $13,5 \mathrm{~g} / \mathrm{dl}$, neutrófilos $20,4 \%$, linfocitos $69,2 \%$, monocitos $9.19 \%$, eosinófilos $0,48 \%$, basófilos $0,77 \%$. Dado el buen estado clínico del paciente y laboratorios normales, no ameritó cobertura antibiótica. Se mantiene en observación por 48 horas, sin nuevos picos febriles, en buenas condiciones generales, sin alteraciones cardiorrespiratorias u otro signo al examen clínico. Presentó aumento del gasto fecal, sin moco ni sangre. Tolerando vía oral por succión con leche materna y formula. Se recibe reporte a los siete días de su egreso de RT-PCR tiempo real para SARS-CoV2 (COVID 19) protocolo Berlín-2020 positivo.

\section{Discusión}

El caso reportado, concordante con la literatura, evidencia la presentación de síntomas típicos en el periodo neonatal de la infección por SARS-CoV2, como fiebre. Los síntomas respiratorios del paciente no fueron documentados en nuestra institución, y quedan dudas de las versiones del sitio remisión y de la madre. Dada la discrepancia, es posible que en épocas de pandemia como la actual, la realización de las pruebas sea la única manera de detectar posibles casos. Los síntomas gastrointestinales sí fueron documentados durante su estancia. Estos ya habia sido reportados en el país con clínica de sepsis, disfunción hepática, hematológica y sospecha de enterocolitis. Además, se deben de considerar los neonatos positivos como una forma indirecta de detección de casos domiciliarios asintomáticos ${ }^{6}$. Desafortunadamente los familiares rechazaron la prueba. Situación ya reportada, posiblemente por la estigmatización de esta enfermedad $\mathrm{y}$ temores alrededor de la misma ${ }^{7}$.

La mayoría de la literatura se centra en los hijos de madres positivas para SARS-CoV2 al momento del nacimiento, por el potencial riesgo de transmisión horizontal. Sin embargo, cada vez hay más reportes similares al nuestro, en los cuales la infección se da a través de contactos domiciliaros con síntomas leves o asintomáticos para SARS-CoV2 y los recién nacidos detectados positivos ayuden a la deteccion de nuevos casos asintomáticos o leves, buscando determinar la fuente de contagio ${ }^{6,8}$. Además, por la fecha de aparición de síntomas, la probabilidad de trasmisión horizontal por contactos domiciliarios es la más probable. El periodo de incubación de la infección está entre 5-7 días, pudiendo ir hasta $14^{8}$. Por lo cual se hace poco probable la trasmisión vertical, que continua siendo tema de debate. Aunque es probable y plausible, dado el reporte de otros HCoVs con esta forma de trasmisión?.

Los síntomas en el periodo neonatal son inespecíficos y normalmente tienen un contacto epidemiológico positivo, usualmente la madre, que orienta al diagnóstico 5 .

La fiebre constituye un signo cardinal en muchos de los reportes, incluso como único síntoma en un 50\% de los casos y está asociado en un gran número de casos, como el nuestro. Otros síntomas pueden estar asociados en menor frecuencia como letargia, síntomas respiratorios de diferente expresión cínica (rinorrea, tos, quejido, aspecto moteado, apnea y dificultad respiratoria) y síntomas gastrointestinales (rechazo a la vía oral, vómito y distensión abdominal), estos últimos relevantes en edad neonatal. Ver tabla ${ }^{6,10-19}$. En neonatos con factores de riesgos como inmunodeficiencia, cardiopatías congénitas, displasia broncopulmonar y malformación del tracto respiratorio, los hallazgos clínicos deben ser analizados cuidadosamente ${ }^{5,7}$. La hipoxemia que ha sido el principal punto de preocupación en la edad adulta, no es reportado en la población neonatal, como un signo clinico importante.

Si bien, el principal enfoque ha sido en demostrar la trasmisión vertical, la trasmisión horizontal está siendo reportada cada vez más frecuente. Con manifestaciones clínicas muy sutiles, e incluso asintomáticos, que nos deben motivar a tomar más pruebas en este grupo etario ${ }^{20}$.

La trasmisión de forma horizontal se da a través de contacto estrecho (madre o cuidador infectado) por diseminación de gotas cuando una persona tose, estornuda o habla. Además, el virus puede permanecer en superficies por largo tiempo, lo que genera riesgo adicional al tocar superficies infectadas, y luego tocarse los ojos o cara con las manos contaminadas por el virus. Adicionalmente, SARS-CoV2 ha sido encontrado en muestra fecal de algunos pacientes en Estados Unidos y China, por lo cual la posibilidad de trasmisión orofecal no podría ser descartada ${ }^{21}$. En casos de síntomas gastrointestinales su búsqueda del virus en materia fecal podría ser una opción. 
Este virus RNA tiene una alta capacidad de mutaciones, lo que lo hace capaz de evadir el sistema inmune y generar nuevas infecciones. Parece que actúa por la interacción de la una proteína viral (spine protein) con receptores de la enzima convertidora de angiotensina (ACE2). El SARS-CoV 1 y 2 tienen alta afinidad por los receptores ACE2, siendo la afinidad de SARS-CoV2 10-20 veces mayor. Esta interacción es la responsable de la replicación viral, y las manifestaciones clínicas están en relación con las células que expresan los receptores ACE2. Estos se encuentran a nivel pulmonar, intestinal, renal y en vasos sanguíneos. La interacción del SARS-CoV2 con estos receptores modula negativamente su expresión, lo cual parece ser responsable del daño sistémico, principalmente a nivel pulmonar. El daño generado por la interacción viral y los receptores ACE2, es secundario a que estos últimos son disparadores de procesos inflamatorios a nivel sistémico. Esto explicaría los síntomas propios de la infección y el menor impacto en periodo neonatal, al menos de forma teórica. Otras hipótesis están en relación con la inmadurez de los receptores ACE2 en el periodo neonatal $1^{4,8,15,19,22}$. Estudios recientes han demostrado que estos receptores se incrementan con la edad, lo que explicaría aún la menor severidad en el periodo neonatal ${ }^{23}$. Otros mecanismos que podrían estar involucrados son la mayor relación de linfocitos CD4 - CD8 en neonatos. Esto ha sido reportado, en niños mayores con infección por SARS-CoV2, donde hay niveles más bajo de conteos de $\mathrm{CD} 4{ }^{15}$.

El abordaje dependerá de la expresión clínica pero hemograma, pruebas de coagulación, pruebas de función renal, hepática, radiografía de tórax serán necesarias, y otras según manifestaciones clínicas. Pero lo más importante será la búsqueda del virus mediante RT-PCR en vías respiratorias, sangre e incluso materia fecal $^{4}$. Esta última puede tener implicaciones en la forma de trasmisión y prevención de diseminación.

Si bien, el resultado de los casos reportados hasta la fecha, son de buena evolución, el impacto puede ser diferente en otros grupos de poblaciones con factores de riesgos, como pueden ser prematuros, neonatos con cardiopatías congénitas, anemia, desnutrición, bajo peso, displasia broncopulmonar y malformaciones de la vía área entre otras. Por lo que evaluar contactos estrechos de este grupo de riesgo debe ser prioridad al momento del egreso.

Por otra parte, se conoce poco sobre el impacto de estas infecciones a mediano y largo plazo. Los estudios de seguimiento podrían ser necesarios.
Para finalizar, las trasmisión horizontal es posible, e incluso podría ser el primer eslabón de casos domiciliarios asintomáticos o sintomáticos leves. Por otro lado, la expresión clínica variable de la enfermedad obliga al estudio de neonatos febriles, que parece ser importante en este momento de pandemia.

\section{Referencias}

1. 1er Consenso colombiano de atención, diagnóstico $\mathrm{y}$ manejo de la infección por SARS-COV-2/ COVID-19 -19 en establecimientos de atención de la salud : Infectio; 2020.

2. Sisman J, Jaleel M, Moreno W, Rajaram V, Collins $\mathrm{R}$, Savani R, et al. Transmisión intrauterina de la infección por SARS-COV-2 en un lactante premature. Pediatr Infect Dis J. 2020; 39(9): e265-e267. doi: 10.1097/INF.0000000000002815

3. Vivanti AJ, Vauloup-fllous C, Prevot S, Zupan V, Suffee C, Do Cao J; et al. Transplacental transmission of SARS-CoV-2 infection. Nat Commun. 2020; 11(1): 1-7. doi: 10.1038/s41467-020-17436-6

4. Instituto nacional de salud. Ministerio de Salud Gobierno de el Salvador. https://www.salud.gob.sv/ boletines-epidemiologicos-2018/?wpdmc=boletinesepidemiologicos-ano-2018.

5. Ovali, F. SARS-CoV-2 Infection and the Newborn. Front Pediatr. 2020; 8(1): 1-17. doi: 10.3389/ fped.2020.00294

6. Baquero H, Vanegas ME, Velandia L, Neira F, Navarro E. Sepsis neonatal tardía por SARS CoV2. Biomédica. 2020; 40(2): 1-6. doi: 10.7705/ biomedica.5609

7. De rose DU, Piersigilli F, Ronchetti MP, Santisi A, Bersani I, Dotta A, et al. Novel Coronavirus disease (COVID-19) in newborns and infants: what we know so far. Ital J Pediatr. 2020; 46(56): 1-8. doi: 10.1186/s13052-020-0820-X

8. Meslin P, Guiomard C, Choukria MP, Porcher J, Duquesne F, et al. Coronavirus Disease 2019 in Newborns and very young infants: a series of six patients in France. Pediatr Infect Dis. 2020; 39(7): e145-147. doi: 10.1097/ INF.0000000000002743

9. Peng Z, Wang J, Mo Y, Duan W, Guangjun X, et al. Unlikely SARS-CoV-2 vertical transmission from mother to child: A case report. J Infect Public Health.2020; 13(5): 818-820. doi: 10.1016/j. jiph.2020.04.004

10. Zeng LK, Tao XW, Yuan WH, Wang J, Liu X, Liu ZS. First case of neonate infected with novel coronavirus pneumonia in China (abstract). Zhonghua Er Ke Za Zhi.2020;58(0): E009. doi: 10.3760/ cma.j.issn.0578-1310.2020.0009 
11. Zhang ZJ, Xue-Jie Y, Fu T, Yu L, Jiang Y, Xiang-Yang $\mathrm{B}$, et al. Novel coronavirus infection in newborn babies under 28 days in China. Eur Resp J. 2020; 55(6): 1-11. doi: 10.1183/13993003.00697-2020

12. AghdamMK,JafariN,EftekhariK.Novelcoronavirus in a 15-day-old neonate with clinical signs of sepsis, a case report. Infectious Dis (Lond). 2020; 52(6): 427-429. doi: 10.1080/23744235.2020.1747634

13. Buonsenso D, Costa S, Sanguinetti MP, Cattani P, Posteraro B, Marchetti S, et al. Neonatal late onset infection with severe acute respiratory syndrome Coronavirus 2. Am J Perinatol. 2020; 37(8): 869872. doi: $10.1055 / \mathrm{s}-0040-1710541$

14. Patek P, Corcoran J, Adams L, Khandhar P. SARSCoV-2 Infection in a 2-Week-Old Male With Neutropenia. Clin Pediatr. 2020; 59(9-10): 918-920. doi: 10.1177/0009922820920014

15. Coronado Munoz A, Nawaratne U, Mcmann D, Ellsworth M, Meliones J, Boukas K. Late-onset neonatal sepsis in a patient with Covid-19. N Engl J Med. 2020; 382(19): e49. doi: 10.1056/ NEJMc2010614

16. Dumpa V, Kamity R, Vinci AN, Noyola E, Noor A. Neonatal Coronavirus 2019 (COVID-19) Infection: A case report and review of literature. Cureus. 2020; 12(5): e8165. doi: 10.1097/inf.0000000000002752

17. Wardell H, Campbell JI, Vanderpluym C, Dixit A. severe acute respiratory syndrome coronavirus 2 infection in febrile neonates. J Pediatric Infect Dis Soc. 2020; piaa084. doi: 10.1093 / jpids / piaa084

18. White AC, Mukherjee P, Stremming J, Sherlock LG, Reynolds RG, Smith D, et al. Neonates hospitalized with community-acquired SARS$\mathrm{CoV}-2$ in a Colorado neonatal Intensive Care Unit. Neonatology. 2020; 1-5. doi: $10.1159 / 000508962$

19. González Brabin A, Iglesias bouzas MI, Nieto Moro M, Martínez de Azagra-Garde A, García Salido A. Apnea neonatal como manifestación inicial de infección por SARS-CoV-2. An Pediatr. 2020; 93(3): 1-2. doi: 10.1016/j.anpedi.2020.05.008

20. Reddy Kallem V, Sharma D, Nieto Moro M, Martínez de Azagra-Garde A, García Salido A. COVID 19 in neonates. J Matern-Fetal Neonatal Med. 2020: 1-10. doi: 10.1016/j.anpedi.2020.05.008

21. Kant Dhir S, Kumar J, Meena J, Kumar P. Clinical features and outcome of SARS-CoV-2 infection in neonates. J Trop Pediatr. 2020; fmaa059. doi: 10.1093/tropej/fmaa059
22. Zhang W, Hui du R, Li B, Shuang Zheng X, Lou yang $\mathrm{X}$, Ben $\mathrm{H}$, et al. Molecular and serological investigation of 2019- $\mathrm{nCoV}$ infected patients: implication of multiple shedding routes. Emerg Microbes Infect. 2020;9(1): 386-389. doi: $10.1080 / 22221751.2020 .1729071$

23. Yamamoto L, Dos Santos EH, Santos Pinto L, Cisotto Rocha M, Aparecida Kanunfre K, et al. SARS-CoV-2 infections with emphasis on pediatric patients: a narrative review. Rev Inst Med Trop Sao Paulo. 2020; 62: 1-12. doi: 10.1590/ s1678-9946202062065

24. Baruch Steinman J, Moon Lum F, Pui Kay Ho P, Kaminski N, Steinman L. Reduced development of COVID-19 in children reveals molecular checkpoints gating pathogenesis illuminating potential therapeutics. Proc Natl Acad Sci U S A. 2020; 117(40): 1-7. doi: 10.1073/pnas.2012358117 